\title{
Signal Processing of Ground Penetrating Radar Using Spectral Estimation Techniques to Estimate the Position of Buried Targets
}

\author{
Shanker Man Shrestha \\ Arai Laboratory, Electronic Engineering Department, The University of Electro-Communications, \\ 1-5-1 Chofugaoka, Chofu, Tokyo 182-8585, Japan \\ Email:shanker@spica.ee.uec.ac.jp \\ Ikuo Arai \\ Arai Laboratory, Electronic Engineering Department, The University of Electro-Communications, \\ 1-5-1 Chofugaoka, Chofu, Tokyo 182-8585, Japan \\ Email: arai@spica.ee.uec.ac.jp
}

Received 29 March 2003 and in revised form 28 June 2003

\begin{abstract}
Super-resolution is very important for the signal processing of GPR (ground penetration radar) to resolve closely buried targets. However, it is not easy to get high resolution as GPR signals are very weak and enveloped by the noise. The MUSIC (multiple signal classification) algorithm, which is well known for its super-resolution capacity, has been implemented for signal and image processing of GPR. In addition, conventional spectral estimation technique, FFT (fast Fourier transform), has also been implemented for high-precision receiving signal level. In this paper, we propose CPM (combined processing method), which combines time domain response of MUSIC algorithm and conventional IFFT (inverse fast Fourier transform) to obtain a super-resolution and high-precision signal level. In order to support the proposal, detailed simulation was performed analyzing SNR (signal-to-noise ratio). Moreover, a field experiment at a research field and a laboratory experiment at the University of Electro-Communications, Tokyo, were also performed for thorough investigation and supported the proposed method. All the simulation and experimental results are presented.
\end{abstract}

Keywords and phrases: FFT, GPR, MUSIC algorithm, SFCW radar, super-resolution signal processing.

\section{INTRODUCTION}

Spectral estimation techniques have been approved as a unique tool for signal and image processing of radar. There are different spectral estimation techniques, in which conventional fast Fourier transform (FFT) has been widely used for real-time measurement due to higher-computational efficiency and its ability to produce high-precision receiving signal level for a large class of signal processes. However, there are several inherent performance limitations of the inverse fast Fourier transform (IFFT) approach like low-frequency range, that is, its ability to distinguish the spectral response of two or more signals is very low, and implicit windowing of the data, that is, energy of the main lobe of a spectral response leaks into the side lobes.

Generally, ground penetration radar (GPR) is a narrow bandwidth device and its radar range is normally high, a wide bandwidth is greatly desired to enclose all target images, which is difficult to make because it is limited by antenna size in the low-frequency range and underground propagation characteristics in the high-frequency range $[1,2]$. In order to overcome these problems, improvement of frequency resolution is greatly desired. Moreover, improvement of resolution is very important for GPR to trace out closely buried targets, like gas pipes, water pipes, cables, and so forth, in an urban area and also to detect the buried land mines that cause thousands of human life every year throughout the world $[3,4]$. Therefore, we implemented super-resolution spectral estimation technique multiple signal classification (MUSIC) algorithm to improve the resolution capacity. Also, we implemented conventional FFT to obtain the high-precision signal level.

Several algorithms for super-resolution spectral estimation have been proposed, for example, maximum likelihood method (MLM) [5], minimum entropy method (MEM) [6], estimation of signal parameter via rotational invariance technique (ESPRIT) [7], MUSIC [8], and so forth. In this paper, the MUSIC algorithm, proposed by Schmidt [8], which 


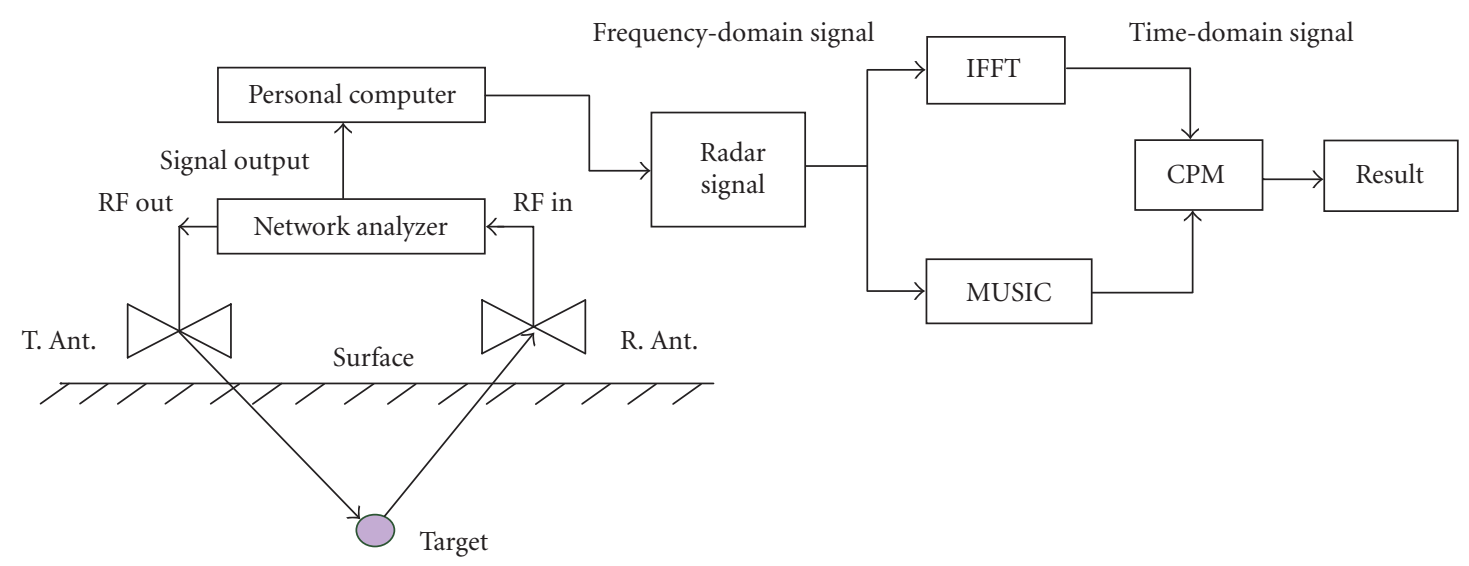

FIGURE 1: Simple block diagram of radar system and proposed signal processing technique.

requires preprocessing to decorrelation is used as a reference. Schmidt's approach yields high resolution even if the signals are partially correlated but does not cover the decorrelation technique. Later, several authors have come up with successful approaches to decorrelate the coherent signal. Nevertheless, spatial smoothing process (SSP), which is based on the spatial averaging technique, proposed by Evans [9] and later presented with more complete analysis by Shan [10], has gained wide popularity. Further, a modified form of SSP (MSSP) has been proposed by Williams [11]. We implemented both SSP and MSSP preprocessing techniques for simulation and experimental data processing.

The remainder of this paper is arranged in the following manner. Signal and image processing methodology and necessary formulation are presented in Section 2. Proposal of CPM (combined processing method) and simulation results are presented in Section 3. Field experiment and laboratory experiment results as well as their performance analysis are presented in Sections 4 and 5, respectively. Finally, we conclude this paper in Section 6.

\section{METHODOLOGY AND FORMULATION}

This research deals with a signal processing method used to increase the vertical resolution of a radar image and to obtain a high-precision signal level. Generally, there are two types of GPRs, pulsed radar and FMCW (frequency modulation continuous wave) radar [12]. Pulsed radar operates in the time domain whereas FMCW radar operates in frequency domain. The pulsed radar generates the pulse with a wide frequency spectrum and performs sampling at successive pulse to obtain the signal wave form. In contrast, FMCW radar produces a sinusoidal wave that sweeps through a predefined frequency band and measures the return signal strength at different frequencies to obtain the frequency spectrum of the target return. Therefore, frequency-domain signal can be directly measured from the FMCW radar. Most GPR are in the time domain using the pulse signal, and sensitivity and maximal detectable depth are usually limited by the antenna. Recently, more FMCW GPRs are emerging due to larger dynamic range, less power consumption, and more convenient calibration. In this research work, we used a vector network analyzer as a GPR, which is based on an SFCW (step frequency continuous wave) radar. This SFCW radar operates in the frequency domain and is almost similar to the FMCW radar except that the frequency changes in steps. The vector network analyzer transmits the frequency-domain complex signal which has real and imaginary parts and received the complex-reflected signal at different frequencies. This reflected frequency-domain data is considered as a radar signal [13]. The data acquisition and the signal processing methods, and experiment methodology are shown in Figure 1 and the procedures are explained as follows.

(1) The frequency-domain radar signal spectrum is received and it undergoes IFFT processing to obtain a high-precision receiving signal level.

(2) The same frequency-domain radar signal undergoes MUSIC processing to obtain high time-delay resolution.

(3) Time-domain responses are obtained in both cases. The time-domain responses of IFFT and MUSIC are combined, in a process we call CPM.

(4) A simulation was carried out to verify the proposed method. The signal-to-noise ratio (SNR) was analyzed to investigate the efficiency and the robustness of the proposed method.

(5) A field experiment was performed to support the simulation and to obtain a real image of the buried targets in a soil medium.

(6) A lab experiment was performed to investigate the maximum resolution capacity that can be detected by the proposed method in a water medium.

The objective of this research is to apply the proposed method for the signal processing and image reconstruction of GPR. 


\subsection{MUSIC algorithm}

The MUSIC algorithm is a nonparametric spectral estimation technique, which estimates multiple scattering centers from the observed voltage received on an array of antenna utilizing the eigenvector. The eigenvectors can be used to compute a spectrum with DOA (direction of arrival) $[14,15$, 16] and estimate delay time of high-frequency spectrum [8]. The eigenvalue of diagonal matrix helps to estimate the numbers of reflected signals.

The measured value of reflected signal from the target with a vector network analyzer can be expressed using vector notation as follows:

$$
\mathbf{x}=\mathrm{Ay}+\mathbf{w}
$$

where

$$
\begin{aligned}
\mathbf{x} & \cong\left[x_{1}, x_{2}, \ldots, x_{L}\right]^{T}, \\
\mathbf{A} & \cong\left[\mathbf{a}\left(\boldsymbol{\tau}_{1}\right), \mathbf{a}\left(\boldsymbol{\tau}_{2}\right), \ldots, \mathbf{a}\left(\boldsymbol{\tau}_{L}\right)\right], \\
\mathbf{a}\left(\boldsymbol{\tau}_{K}\right) & \cong\left[e^{-j 2 \pi f_{1} \tau_{L}}, e^{-j 2 \pi f_{2} \tau_{L}}, \ldots, e^{-j 2 \pi f_{k} \tau_{L}}\right]^{T}, \\
\mathbf{y} & \cong\left[y_{1}, y_{2}, \ldots, y_{L}\right]^{T}, \\
\mathbf{w} & \cong\left[w_{1}, w_{2}, \ldots, w_{L}\right]^{T} .
\end{aligned}
$$

Here, $T$ represents transpose. Again $\mathbf{a}(\boldsymbol{\tau})$ vector can be declared by its time, so it is called a mode vector. The symbol $\mathbf{A}$ is a delay parameter matrix which has $L$ numbers of arrays and the $k$ th element of row. So, $L$ can be regarded as the number of signals while the symbol $\mathbf{y}$ is the reflection coefficient of the $L$ th reflection point at frequency $f_{k}$ and $\mathbf{w}$ is a noise vector. The $L L$ signal covariance matrix of $\mathbf{x}$ vector is represented by

$$
\mathbf{S}=\mathbf{x x}^{*}=(\mathbf{A y}+\mathbf{w})(\mathbf{A y}+\mathbf{w})^{*},
$$

where $*$ denotes complex conjugate transpose. Also, arriving wave and internal noise can be considered as not related (orthogonal), and the signal covariance matrix becomes

$$
\mathbf{S} \cong \mathbf{A P A}^{*}+\sigma^{2} \mathbf{I}
$$

Here, the elements of the noise vector $\mathbf{w}$ are mean zero and $\sigma^{2}$ is the variance.

The position (delay time) of each reflection point can be estimated by searching the peak position of the MUSIC function $\left(P_{\text {music }}\right)$

$$
P_{\text {music }}(\boldsymbol{\tau})=\frac{\mathbf{a}(\boldsymbol{\tau})^{*} \mathbf{a}(\boldsymbol{\tau})}{\mathbf{a}(\boldsymbol{\tau})^{*} \mathbf{E}_{N} \mathbf{E}_{N}^{*} \mathbf{a}(\boldsymbol{\tau})},
$$

where $\mathbf{a}(\boldsymbol{\tau})$ is a delay-time mode vector and $\mathbf{E}_{N}$ is the noise $L(L-k)$ matrix whose columns are the $(L-k)$ noise eigenvector.

\subsection{Smoothing process}

GPR signals are generally coherent signals as the measurements was taken by SFCW radar based on vector network
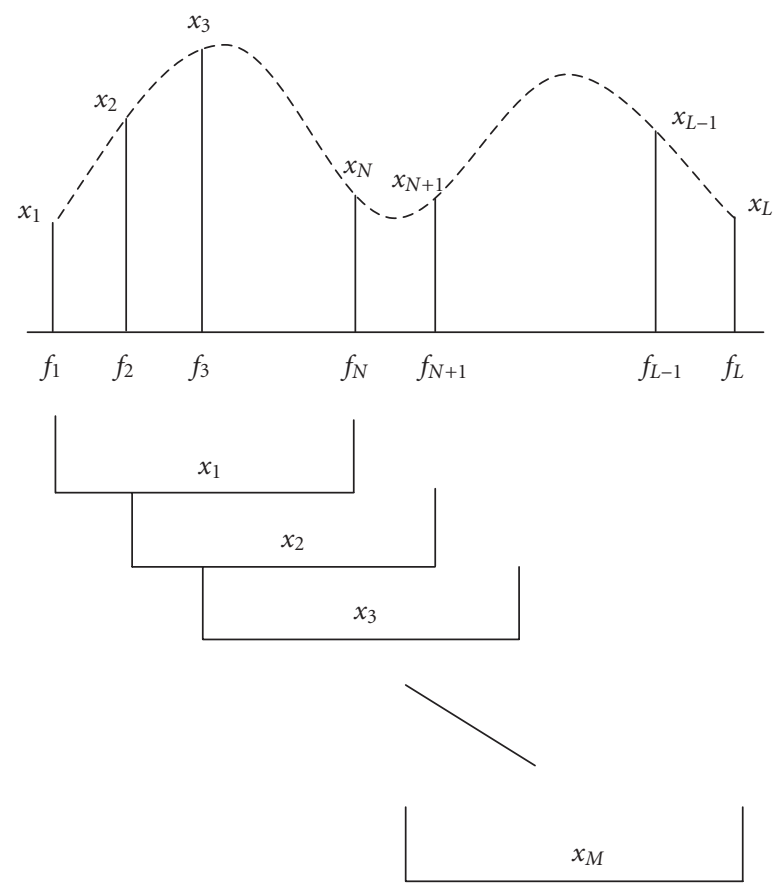

FIgURE 2: Frequency-domain subarray arrangement.

analyzer. The vector network analyzer generates the identical signal, and the phase and the amplitude of the reflected signals also do not change from snapshot to another. MUSIC fails to work properly when the signals are coherent. So, a decorrelation process is performed in order to eliminate the problems encountered with coherent signals. The received signal is divided into the numbers of overlapping subarrays or snapshots as shown in Figure 2. Consequently, the phase is changed in each snapshot. Two smoothing methods have been proposed so far, SSP and MSSP.

\subsubsection{Spatial smoothing process (SSP)}

We consider the frequency-domain array with $L$ reflection coefficient that extends from $(1,2,3, \ldots, N, N+1, \ldots, L-$ $1, L)$, making $M$ number of overlapping snapshots having length $N$, as shown in Figure 2. The relation between $L, M$, and $N$ can be formulated as

$$
L=N+M-1
$$

Let $x_{1}$ be the first snapshot having length $N$ and $x_{2}$ the second snapshot and extending up to $x_{M}$ overlapping snapshot. The phase changes in each snapshot. We have the Lth element of subarray in our model equation, so it can be written as

$$
\mathbf{x}_{k}=\mathbf{A D}^{(k-1)} \mathbf{y}+\mathbf{w}_{k}
$$

where $\mathbf{D}^{k}$ denotes the $k x k$ diagonal matrix, represented as

$$
\mathbf{D}=\operatorname{diag}\left\{e^{-j 2 \pi \Delta f \tau_{1}}, e^{-j 2 \pi \Delta f \tau_{2}}, \ldots, e^{-j 2 \pi \Delta f \tau_{k}}\right\},
$$

where $\Delta f$ is the sampling frequency separation. 
TABLE 1: Parameter setting for simulation.

\begin{tabular}{ll}
\hline Center frequency & $85 \mathrm{MHz}$ \\
$Q$ factor & 1 \\
Frequency bandwidth & 125 \\
Number of signals & 3 \\
Delay separation of each signal & $20 \mathrm{~ns}$ \\
Sampling frequency & $1 \mathrm{MHz}$ \\
\hline
\end{tabular}

The covariance matrix of the $k$ th subarray is given by

$$
\begin{gathered}
\mathbf{S}_{k}=\mathbf{x}_{k} \mathbf{x}_{k}^{*}, \\
\mathbf{S}_{k}=\left(\mathbf{A D}^{(k-1)} \mathbf{y y}^{*} \mathbf{D}^{(k-1)^{*}} \mathbf{A}^{*}\right)+\boldsymbol{\sigma}^{2} \mathbf{I} .
\end{gathered}
$$

The spatially smoothed covariance matrix is defined as the sample means of the subarray covariance and is expressed as

$$
\mathbf{S}_{\mathrm{SSP}}=\frac{1}{M} \sum_{k=1}^{M} \mathbf{S}_{k} .
$$

\subsubsection{Modified spatial smoothing process (MSSP)}

In MSSP, the covariance matrix is expressed as

$$
\mathbf{S}_{k}=\mathbf{J} \mathbf{S}_{k}^{*} \mathbf{J}
$$

where $\mathbf{J}$ denotes an $N \times N$ exchange matrix and is formulated as follows

$$
\mathbf{J}=\left[\begin{array}{ccccc}
0 & 0 & \cdots & 0 & 1 \\
0 & 0 & \cdots & 1 & 0 \\
\vdots & \vdots & . & \vdots & \vdots \\
0 & 1 & \cdots & 0 & 0 \\
1 & 0 & \cdots & 0 & 0
\end{array}\right]
$$

Equation (10) is equivalent to

$$
\mathbf{S}_{k}=\left(\mathbf{J A D}^{(k-1)} \mathbf{y} \mathbf{y}^{*} \mathbf{D}^{(k-1)^{*}} \mathbf{A}^{*} \mathbf{J}\right)+\boldsymbol{\sigma}^{2} \mathbf{I} .
$$

Here,

$$
\begin{gathered}
\mathbf{J A}=\mathbf{A} \mathbf{D}^{(N-1)}, \\
\mathbf{S}_{k}=\mathbf{A D}^{(N+k-2)} \mathbf{y y}^{*} \mathbf{D}^{(N+k-2)^{*}} \mathbf{A}^{*}+\boldsymbol{\sigma}^{2} \mathbf{I} .
\end{gathered}
$$

Performing spatial averaging, MSSP can be expressed as

$$
\mathbf{S}_{\mathrm{MSSP}}=\frac{1}{2 \mathbf{M}} \sum_{k=1}^{M}\left(\mathbf{S}_{k}+\mathbf{J} \mathbf{S}_{k} \mathbf{J}\right)
$$

In MSSP, when the effective band is reduced from $L$ to $N$, the resolution will be reduced; nevertheless, the resolution is far better than with IFFT. In other words, if $M$ is increased, $N$ will be decreased because $L=N+M-1$. Consequently, decorrelation performance is increased and reso- lution is decreased. On the other hand, if $M$ is decreased, $N$ will be increased, which means that the effective bandwidth will be increased and the resolution will also be increased but the decorrelation performance will be degraded. The minimum value $M$ gives range profiles of high resolution but some dominant scattering center may not be detected due to the degradation of decorrelation performance and vice versa [17]. Therefore, $M$ should be adjusted according to the nature of target recognition.

\section{SIMULATION}

We generate a radar signal using a bandpass filter (BPF) which can be expressed by

$$
H(f)=\frac{j\left(\omega_{\circ} / Q\right) \omega}{\left(\omega_{\circ}^{2}-\omega^{2}\right)+j\left(\omega_{\circ} / Q\right) \omega},
$$

where $\omega=2 \pi f, Q=1$, and $f_{0}=85 \mathrm{MHz}$. Parameters for the simulation are shown in Table 1.

The BPF generates complex frequency-domain data having real and imaginary information. This frequency-domain spectrum of radar data, shown in Figure 3a, undergoes IFFT processing. In the mean time, the same radar signal undergoes MUSIC processing and the results are comparatively studied $[18,19]$. The frequency-domain data is converted into the time-domain data in both cases. Let the complex IFFT results in the time domain be represented by $X(t)$ and the MUSIC results $Y(t)$. The time-domain results of IFFT and MUSIC are combined using the CPM, which is calculated using the following expression:

$$
Z(t)=\frac{X|\partial Y / \partial t|+Y|\partial X / \partial t|}{\sqrt{|\partial Y / \partial t|^{2}+|\partial X / \partial t|^{2}}}
$$

where $Z(t)$ is the time-domain data of CPM.

In order to explain (18), mathematical analysis has been performed. Since combining the time-domain responses of IFFT and MUSIC are performed by calculating the slope and the position of the signal, the delay of the IFFT response signal and MUSIC response signal should be coincided, which is a required condition. So, in this particular condition, when we consider the point at the peak (centre) of the curve (Figure $3 \mathrm{~b}$ ), the slope of MUSIC will be very higher than the slope of IFFT due to sharp response of MUSIC. This can be expressed mathematically by

$$
\frac{\partial Y}{\partial t} \gg \frac{\partial X}{\partial t} \quad \text { so that } \frac{\partial X}{\partial t} \geq 0 .
$$

Substituting the value of $\partial X / \partial t$ in (18) gives

$$
Z(t)=X
$$

Equation (20) means that the signal level of $Z(t)$ will be the amplitude of the IFFT. Similarly, when we consider the point drifted from the peak (center) of the curve (Figure 3b), 


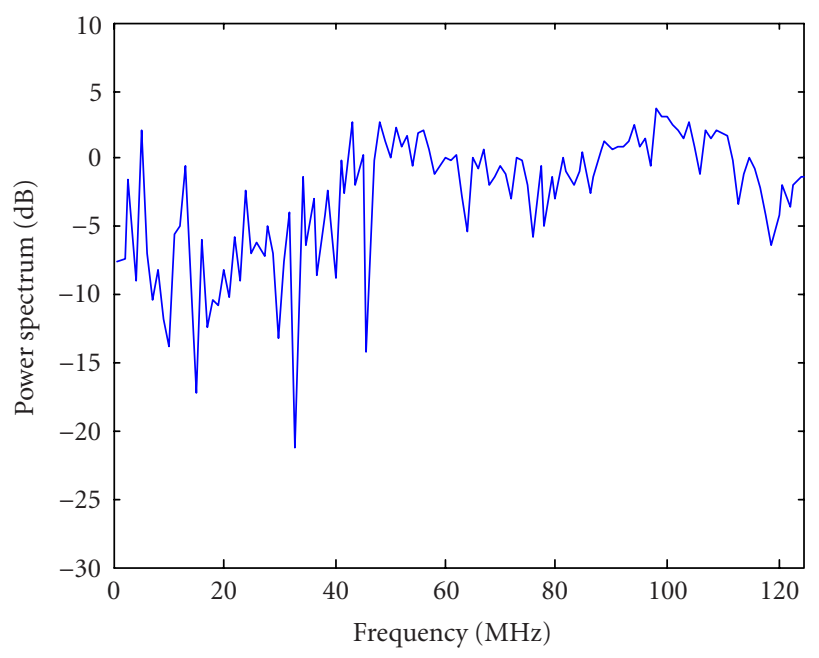

(a) Frequency spectrum of radar signal.

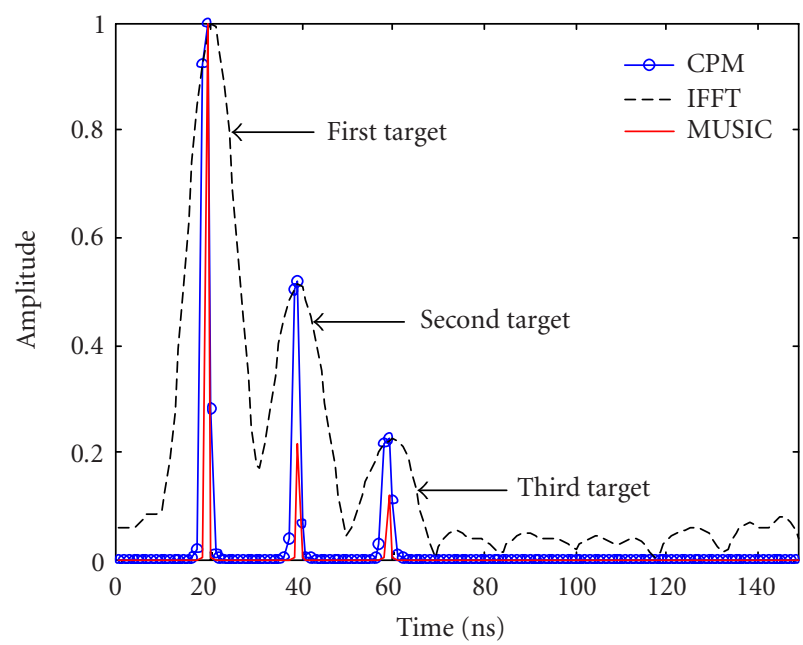

(b) IFFT, MUSIC, and CPM responses.

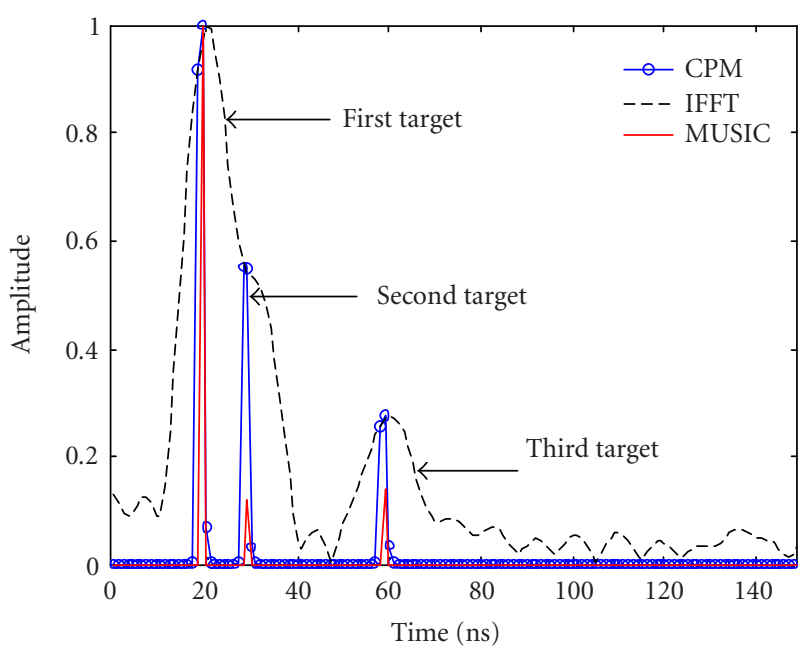

(c) Demonstration of CPM response to resolve the close targets.

FIGURE 3: Simulation result to demonstrate the IFFT, MUSIC, and CPM responses at bandwidth $=125 \mathrm{MHz}, Q=1$, sampling point $=125$, sampling frequency $=1 \mathrm{MHz}$, number of snapshots $(M)$ $=222$.

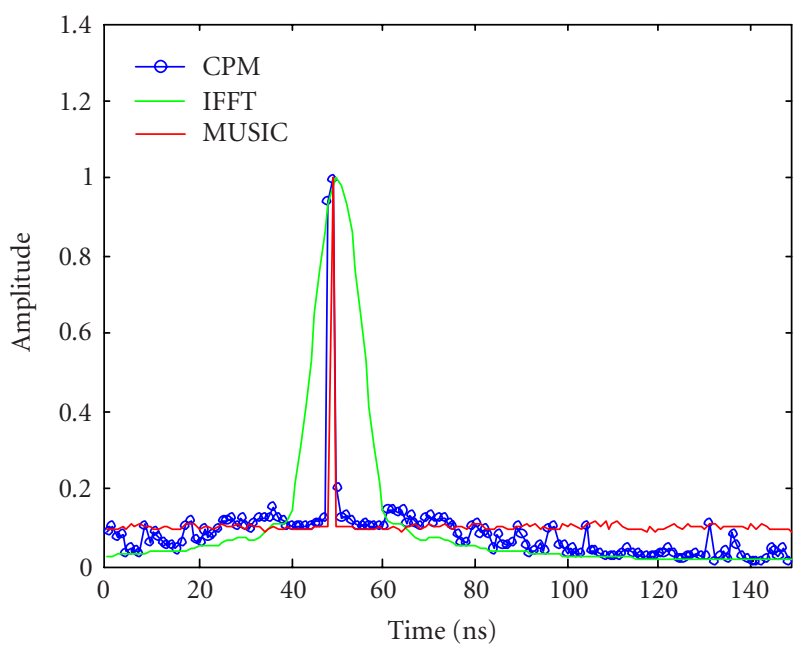

(a) IFFT, MUSIC, and CPM responses of signal only.

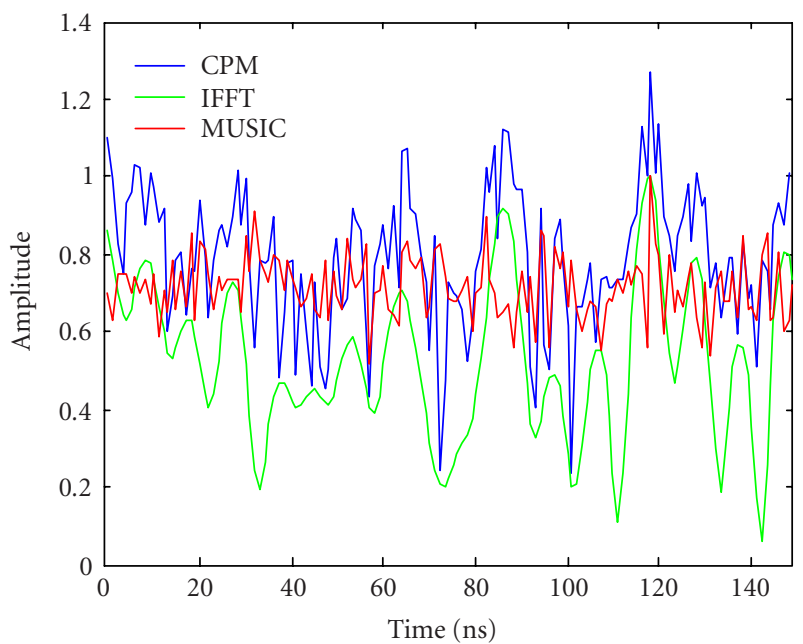

(b) IFFT, MUSIC, and CPM responses of noise only.

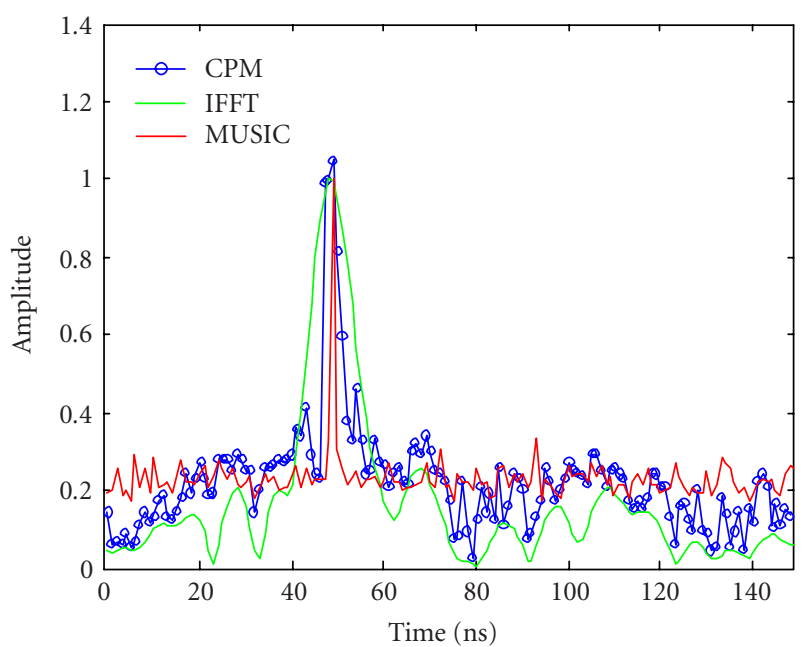

(c) IFFT, MUSIC, and CPM responses of signal plus noise.

FIGURE 4: Simulation results of input SNR $-5.8 \mathrm{~dB}$ and $M=30$. (a) IFFT, MUSIC, and CPM responses of signal only. (b) IFFT, MUSIC, and CPM responses of noise only. (c) IFFT, MUSIC, and CPM responses of signal plus noise. 
the slope of IFFT response curve is very higher than MUSIC response curve. It can also be expressed mathematically by

$$
\frac{\partial X}{\partial t} \gg \frac{\partial Y}{\partial t} \quad \text { so that } \frac{\partial Y}{\partial t} \geq 0 .
$$

Substituting the value of $\partial X / \partial t$ in (18) gives

$$
Z(t)=Y .
$$

Equation (22) means that the signal level of $Z(t)$ will be the amplitude of the MUSIC. Therefore, it is found that $Z(t)$ selects the precision signal level of $X$ (IFFT response) when the slope of $X$ is low and also selects the signal level of $Y$ (MUSIC response) when the slope of $Y$ is low.

It is observed in Figure $3 \mathrm{~b}$ that the IFFT response gives correct amplitude. The amplitude of the second signal was set to the half of the first signal and the third signal was set to one-fourth of the first signal. The response of the IFFT changed according to the value set. However, resolution is very poor and also suffers from a windowing problem. In MUSIC response, the output signal is very sharp and the resolution is very high as it is estimated from the peak of the MUSIC function. However, the precision of receiving signal level is low. CPM response gives high resolution as well as a high-precision signal level. In this context, high resolution means the maximum resolving capacity between the vertically aligned targets. In Figure 3c, the IFFT could not resolve two closely located targets well, that is, the delay-time difference between the successive signal is 10 nanoseconds. On the other hand, MUSIC could resolve the same two closely located targets well but the precision of signal level is low. However, the proposed method CPM could well resolve two closely located targets with high-precision signal level that is demonstrated in Figure 3c.

\subsection{SNR analysis}

Generally, subsurface radar signals are enveloped by noise when targets are deeply buried. The SNR will be very small because the radar signal decay is caused by both wave spreading and soil absorption, as the wave propagates through the soil. Improvement of SNR is desired to allow a minimum detectable signal to be obtained when investigating deep targets. Practically, minimum detectable signal is based on threshold level which should be set properly, otherwise false alarm might result if the threshold level was set too low, and weak target echo might not be detected if the threshold level was set too high. Therefore, SNR of input signal and each IFFT, MUSIC, and CPM responses have been calculated and analyzed in order to set proper threshold level.

While calculating input SNR, the total energy of the signal and noise is separately summed because the input signal and noise is frequency-domain spectrum. The SNR is given by

$$
\mathrm{SNR}_{\text {input }}=10 \log \frac{\int_{0}^{\infty}\left|F_{s}(f)\right|^{2} d f}{\int_{0}^{\infty}\left|F_{n}(f)\right|^{2} d f}
$$

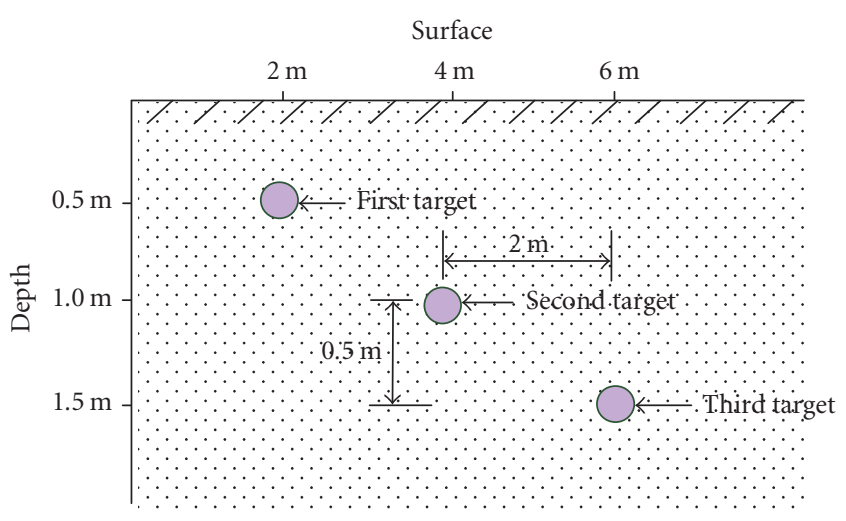

Figure 5: Target position at the experiment field. The target is a steel pipe having diameter of $10 \mathrm{~cm}$.

All the simulations have been performed using Matlab. Noise is generated by a random noise generator function. In order to calculate the output SNR of IFFT response, the IFFT processing has been performed with signal only. Consequently, the time-domain response of signal will be obtained, in which amplitude is measured from zero to peak $\left(V_{0-p}\right)$. Similarly, IFFT is performed with only noise, that is, generated by a random noise generator. As a result, timedomain response is obtained; however, in case of noise, RMS (root mean square) value of all the points of IFFT response should be calculated. SNR of IFFT response of noise is given by

$$
\mathrm{SNR}_{\text {output }}=20 \log \frac{V_{0-p}}{\sqrt{(1 / n) \sum_{i=0}^{n}\left|N\left(t_{i}\right)\right|^{2}}} .
$$

Similarly, SNR of MUSIC and CPM responses are calculated as with IFFT. However, the number of snapshots $M$ was changed while processed by MUSIC to investigate the effect of $M$ with respect to SNR. Simulation results are shown in Figure 4. Input $\mathrm{SNR}$ was $-5.8 \mathrm{~dB}$, the number of overlapping snapshots $M$ was 30 , frequency bandwidth was $250 \mathrm{MHz}$, sampling points was 250, sampling frequency was $1 \mathrm{MHz}$. The comparison is difficult due to great discrepancies observed in the amplitude of IFFT and MUSIC responses. So, MUSIC response has been normalized with IFFT response according to its maximum value of signal level.

In Table 2, the simulation results have been presented varying input SNR. It was found that the minimum detectable signal is $-15.2 \mathrm{~dB}$. The SNR response of IFFT, MUSIC, and CPM at this input SNR has been achieved $35.1 \mathrm{~dB}$, $11.3 \mathrm{~dB}$, and $10.4 \mathrm{~dB}$, respectively. In this case, SNR of IFFT response exhibited superior performance over SNR of MUSIC response. It is observed from the simulation results that $M$ plays an important role in the improvement of SNR and that $M$ varies for smoothing while performing MUSIC processing. When $M$ is increased, SNR will also be improved but resolution will be decreased. On the other hand, decrease in $M$ degrades SNR but increases resolution [17]. This can be 
TABLE 2: Simulation results to investigate the SNR.

\begin{tabular}{ccccc}
\hline Input SNR $(\mathrm{dB})$ & SNR of IFFT response $(\mathrm{dB})$ & No. of snapshots $(M)$ & SNR of MUSIC response $(\mathrm{dB})$ & SNR of CPM response $(\mathrm{dB})$ \\
\hline-15.2 & 35.1 & 30 & 11.3 & 10.4 \\
-5.8 & 45.75 & 30 & 13.6 & 11.1 \\
-5.6 & 45.3 & 235 & 41.5 & 39.8 \\
-5.0 & 45.9 & 100 & 32.1 & 23.6 \\
-6.4 & 46.0 & 75 & 25.2 & 19.8 \\
-4.9 & 49.5 & 50 & 14.2 & 13.4 \\
-4.5 & 50.3 & 30 & 11.5 & 11.7 \\
-2.5 & 53.1 & 30 & 11.9 & 10.5 \\
+2.2 & 57.4 & 30 & 12.6 & 11.8 \\
+7.7 & 63.1 & 30 & 12.9 & 12.0 \\
\hline
\end{tabular}

TABLE 3: Parameter setting for field experiment.

\begin{tabular}{ll}
\hline Frequency bandwidth & $400 \mathrm{MHz}(1-401) \mathrm{MHz}$ \\
Frequency interval & $1 \mathrm{MHz}$ \\
No. of points & 401 \\
Soil conductivity & $0.02 \mathrm{~S} / \mathrm{m}$ \\
Soil permittivity $\varepsilon_{r}$ & 36 \\
Target diameter & $10 \mathrm{~cm}$ \\
Vertical target separation & $50 \mathrm{~cm}$
\end{tabular}

illustrated by the simulation result shown in Table 2 . When $M$ is set to 30 , SNR of MUSIC responses is $13.6 \mathrm{~dB}$ at input $\mathrm{SNR}-5.8 \mathrm{~dB}$ and when $M$ is increased to $100 \mathrm{SNR}$ of MUSIC, the response increases up to $32.1 \mathrm{~dB}$ at input SNR $-5.0 \mathrm{~dB}$. It is to be noted that the SNR of CPM is also increased up to $23.6 \mathrm{~dB}$ due to the effect of MUSIC. Thus, increasing the value of $M$ can increase SNR.

\section{FIELD EXPERIMENT}

A field experiment was performed at the research field of Koden Electronics Company, Yamanashi, Japan, using Network analyzer that has a capability of measuring high-precision data. This vector network analyzer works as an SFCW radar. The experimental field is ordinary soil, a grass landscape with steel pipes buried under the earth at the horizontal separation of about $2 \mathrm{~m}$ and vertical separation of $50 \mathrm{~cm}$, as shown in Figure 5. Parameters for the field experiment are shown in Table 3.

Different sets of experimental data were taken at an increment of $10 \mathrm{~cm}$ to scan the target using ferrite covered bowtie antenna. Frequency-domain data was used to perform IFFT and MUSIC processing simultaneously. Then CPM processing method was performed combining time-domain response of IFFT and MUSIC processing as in simulation process. The comparative study of IFFT, MUSIC, and CPM processing results are shown in Figure 6, when the radar an- tenna is just above the $1 \mathrm{~m}$ depth target. The first signal is the coupling signal between radar antenna and ground. The second signal is target signal.

Two-dimension (2D) and three-dimension (3D) images of IFFT, MUSIC, and CPM are presented in a linear scale, shown in Figure 7. Image of IFFT processing result (Figure 7a) is clear and have continuous diffraction pattern due to the precision receiving signal level. However, resolution could not be considered high, and the impact of windowing and presence of noise could not be ignored. On the other hand, the image of MUSIC processing is sharp and resolution is very high, which is shown in Figure 7c. Moreover, time-side lobe is also significantly eliminated. MUSIC uses eigenanalysis and the number of eigenvalue helps to estimate the number of signal with high resolution. Generally, the eigenvalue below the noise level could be discarded. Eigenanalysis can be performed varying the value of $M$ (snapshot) and $L$ (array element) as explained before in theoretical considerations. Different approaches have been taken to approximate the value of $M$ and $L$ during simulation and experimental data processing as they play a vital role in obtaining good results. In this result (Figure 7c), the value of $M$ is set to 10 and $L$ is set to 50 , the resulting value of $N$ (snapshot length) is set to 41 , which is obtained using (6). The frequency range utilized for MUSIC processing is from $150 \mathrm{MHz}$ to $350 \mathrm{MHz}$.

Finally, the image of CPM processing result in Figure 7e shows that the resolution has been greatly improved from Figure $7 \mathrm{a}$, and the continuity of target image has also been improved from Figure 7c. 3D representation of CPM, shown in Figure 7d, gives clear vision of the targets and shows that the CPM could successfully extract the merits of IFFT and MUSIC algorithm, such as a very high resolution and a very high-precision receiving signal level.

\section{LABORATORY EXPERIMENT}

The encouraging responses with the high resolution and the high-proximity images of the target buried under the 


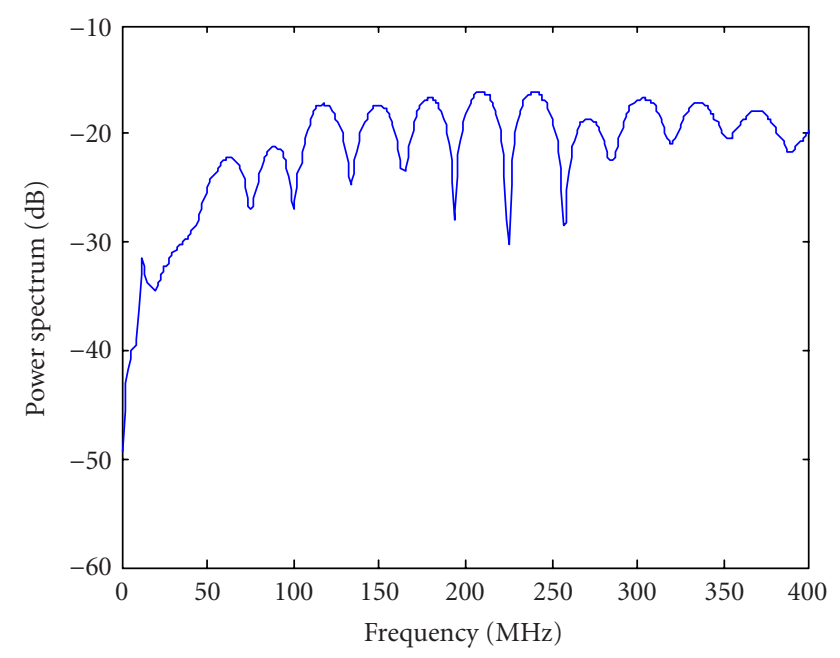

(a) Frequency-domain spectrum of radar signal measured by a vector network analyzer.

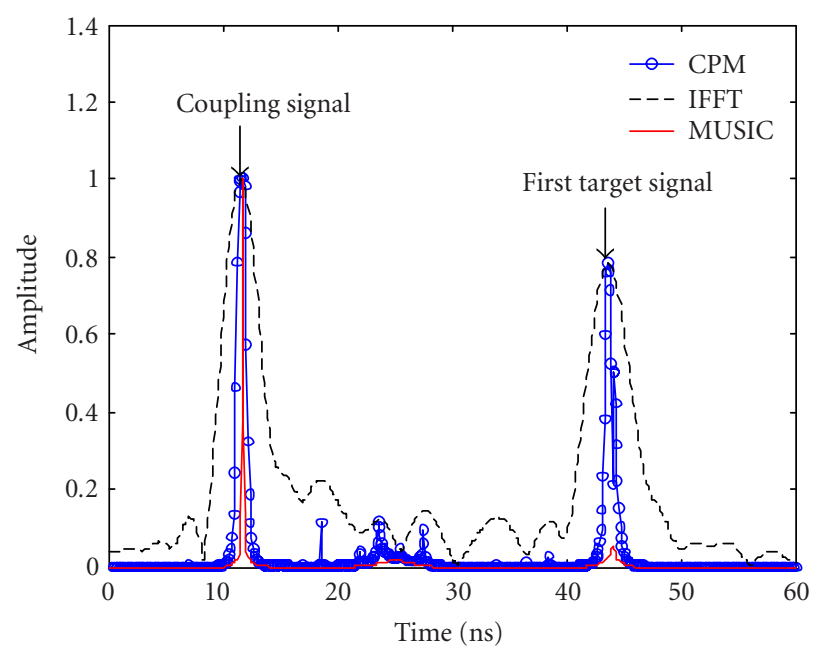

(b) IFFT, MUSIC, and CPM responses of radar data.

FIGURE 6: Experimental result to demonstrate the IFFT, MUSIC, and CPM responses at bandwidth $=400 \mathrm{MHz}$, sampling point $=400$, sampling frequency $1 \mathrm{MHz}$.

soil medium have been demonstrated by the field experiment. Since this research is concentrated to resolve the vertical resolution, further investigation was performed to check the maximum resolution capacity between two vertically aligned targets. For this, experimental setup in the water medium has been developed in the University of ElectroCommunications laboratory with an aquarium of length $40 \mathrm{~cm}$, breadth $40 \mathrm{~cm}$, and height $100 \mathrm{~cm}$. Two targets of copper pipe of $38 \mathrm{~cm}$ long were fixed at the $10 \mathrm{~cm}$ depth from the water surface with a vertical separation of $2 \mathrm{~cm}$ and horizontal separation of $4 \mathrm{~cm}$ as shown in Figure 8.

The experimental setup includes the high-bandwidth antenna, network analyzer, and the signal processing unit. A
TABLE 4: Parameter setting for laboratory experiment.

\begin{tabular}{ll}
\hline Frequency band & $800 \mathrm{MHz}(500-1300) \mathrm{MHz}$ \\
No. of points & 801 \\
Sweep time & $1 \mathrm{~s}$ \\
Small change in frequency & $1 \mathrm{MHz}$ \\
Water relative permittivity $\varepsilon_{r}$ & 81 \\
Target diameter & $1 \mathrm{~cm}$ \\
Vertical target separation & $2 \mathrm{~cm}$ \\
\hline
\end{tabular}

register loaded dipole antenna was used for the experiment. The antenna bandwidth is $600 \mathrm{MHz}$ (500 to $1100 \mathrm{MHz}$ ) and the antenna size was $4 \times 6 \mathrm{~cm}$. Parameter settings in the network analyzer for experimental purposes are as shown in Table 4.

The antenna is submersed in the water; however, water level and top surface of the antenna is kept at the same level. Antenna is moved from left to right at the increment of $1 \mathrm{~cm}$ to scan the target as shown in Figure 8. Different sets of experiment were performed with varying the diameter, material, and the position of the target. Diameter ranges from $1 \mathrm{~cm}$ to $2.5 \mathrm{~cm}$ and material used are aluminum and copper. The vertical target separation ranges from 1 to $10 \mathrm{~cm}$ and the horizontal separation ranges from 0 to $10 \mathrm{~cm}$. These various sets of data have been taken to investigate the magnitude of reflected signal, shadowing effect, and measurements of vertical resolution.

When the diameter of the target is $2.5 \mathrm{~cm}$, the magnitude of the receiving signal is fairly good compared to the target having $1 \mathrm{~cm}$, however, the shadowing effect (the shadow of the upper target) is high when the second target was just below the first one. Similarly, the target at the depth ranging from 10 to $20 \mathrm{~cm}$ results the good receiving signal and increasing target depth will decrease the signal level.

The comparative study of IFFT, MUSIC, and CPM processing results of laboratory experiment are shown in Figure 9, when the radar antenna is just above the first target. The images of laboratory experimental results are shown in Figure 10. Two-dimension imaging of IFFT, MUSIC, and CPM responses are presented in Figures 10a, 10c, and $10 \mathrm{e}$, respectively. Three-dimension imaging of the same IFFT, MUSIC, and CPM responses are presented in Figures $10 \mathrm{~b}, 10 \mathrm{~d}$, and 10f, respectively. All the images are represented in linear scale, and interpolation process has not been performed as in the field experiment as the frequency bandwidth is higher. Kaiser filter was imposed in raw data while performing IFFT processing. MSSP was implemented for MUSIC processing which yield better image than SSP method because of the wide frequency bandwidth data.

The main objective of this experiment is to check the maximum detectable vertical resolution of the proposed method. So, this experiment has been performed with two targets, which are set at the vertical separation of $2 \mathrm{~cm}$. Twodimension and three-dimension images of IFFT response 


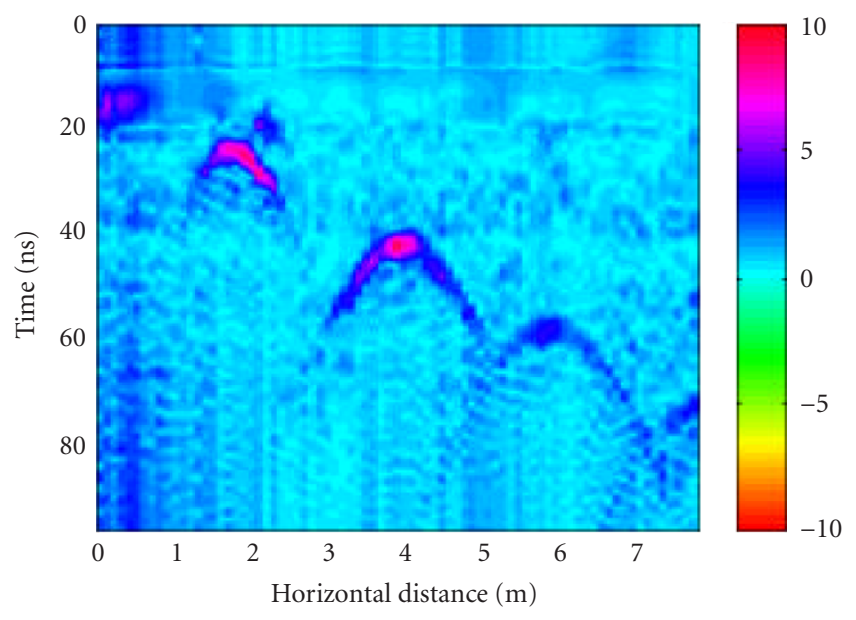

(a) 2D representation of IFFT processing.

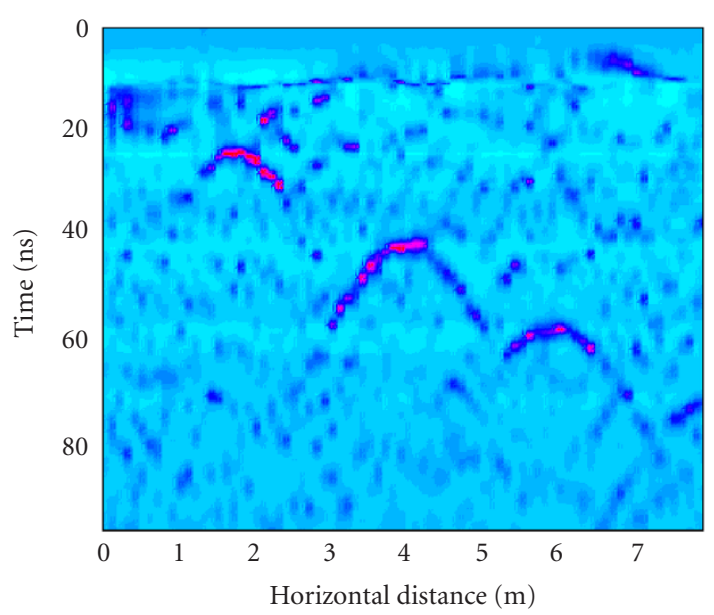

(c) 2D representation of MUSIC processing.

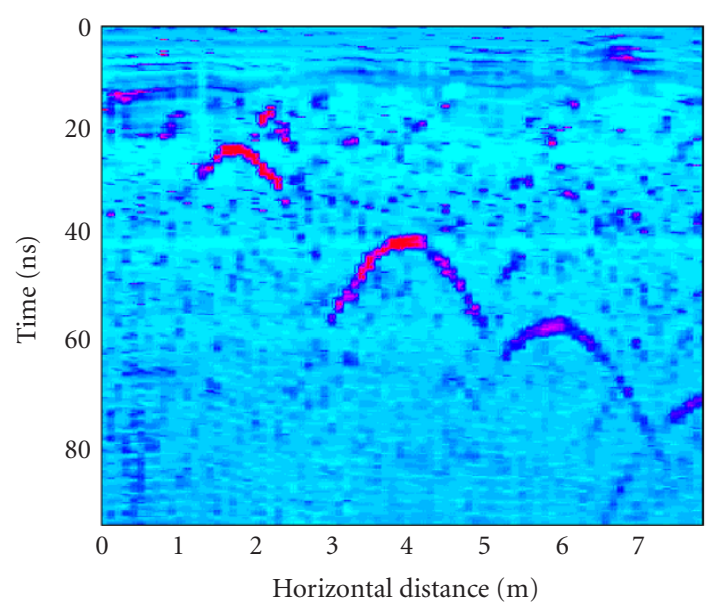

(e) $2 \mathrm{D}$ representation of CPM processing.

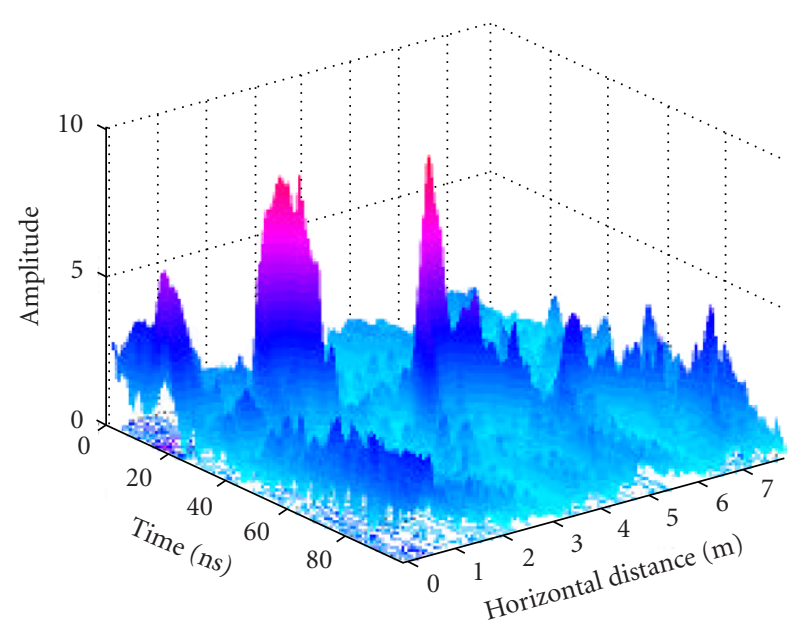

(b) 3D representation of IFFT processing.

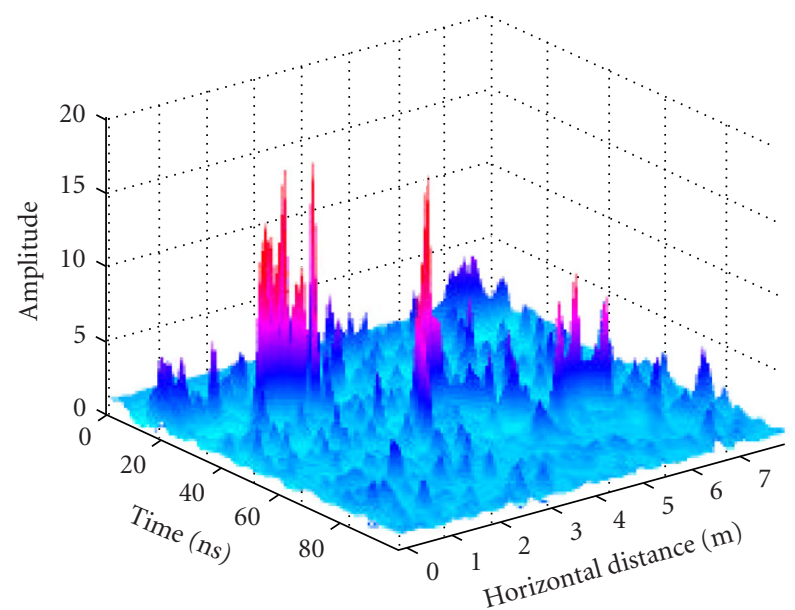

(d) 3D representation of MUSIC processing.

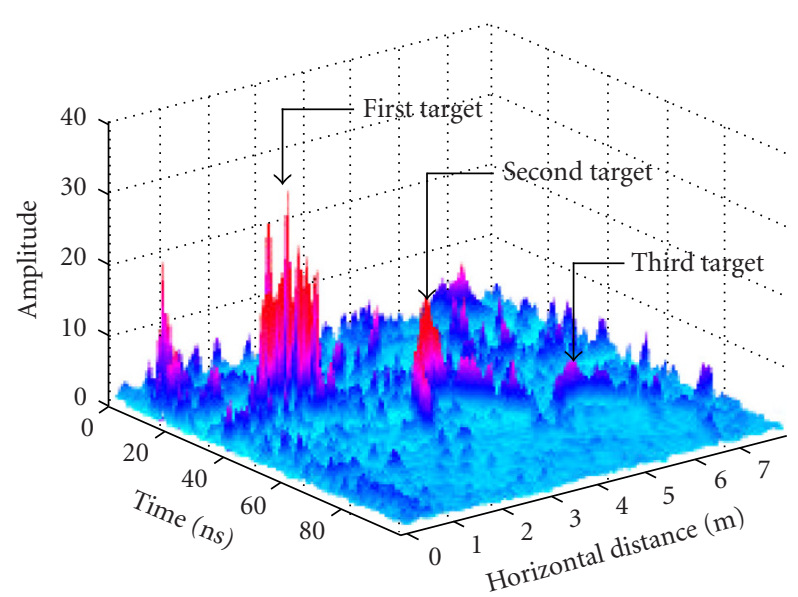

(f) 3D representation of CPM processing.

FIGURE 7: 2D and 3D representations of IFFT, MUSIC, and CPM processing of field experiment data with bandwidth $=400 \mathrm{MHz}, M=10$, and $L=50$. 


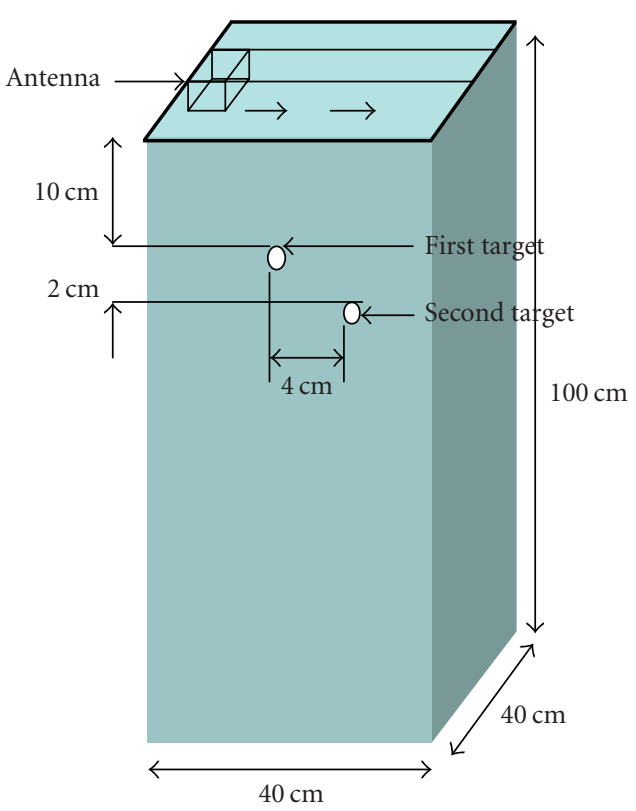

Figure 8: Experiment setup prepared in laboratory using aquarium to do experiment in water medium to check maximum detectable resolution.

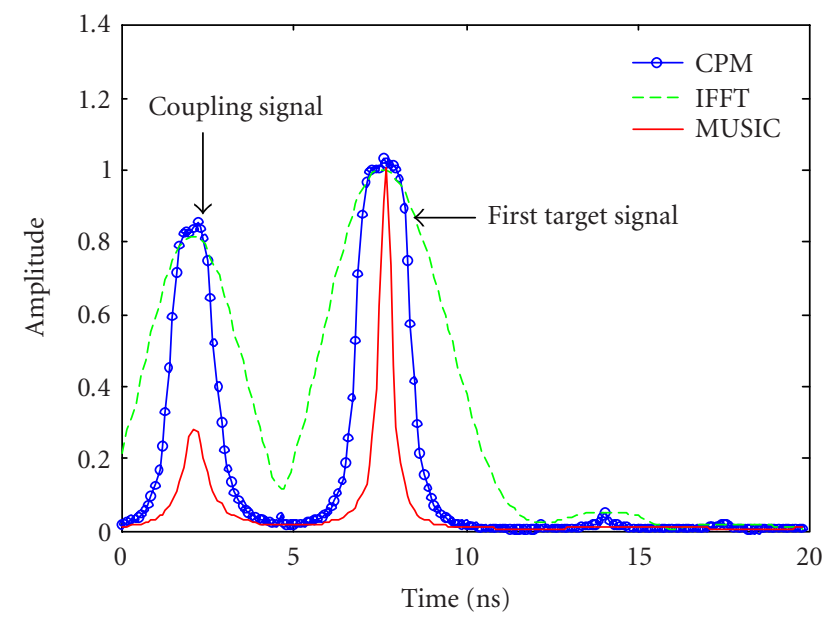

FIGURE 9: IFFT, MUSIC, and CPM responses of laboratory experiment data.

show that the two targets could not be separated by this IFFT processing. If we investigate in an analytic manner, the maximum vertical resolution that can be calculated by FFT is

$$
\Delta r=\frac{c}{2 B \sqrt{\varepsilon_{r}}},
$$

where $\Delta r$ is the vertical resolution between two targets, $c$ is the velocity of light, $B$ is the frequency bandwidth, and $\varepsilon_{r}$ is the relative permittivity of the medium.
In this experiment, (frequency bandwidth) $B$ is set to $800 \mathrm{MHz}$ and (relative permittivity of the water) $\varepsilon_{r}$ is set to 81. Theoretically, IFFT response can resolve a maximum vertical resolution of $2.1 \mathrm{~cm}$ in a noise-free environment. Practically, this could not be achieved due to the presence of noise, leakage of main lobe energy into side lobe, and so forth. This is the very reason that IFFT processing could not resolve the target with the vertical separation of $2 \mathrm{~cm}$. The time-resolution problem could not be overcome in IFFT processing as can be observed in the $3 \mathrm{D}$ imaging of IFFT, shown in Figure 10b. On the other hand, 2D MUSIC response could successfully resolve two or more targets distinctly due to its super-resolution characteristic. 3D presentation in Figure 10d shows a very slim target response without a time-side lobe; however, the continuity of signal could not be maintained. The CPM response, as shown in Figure 10e, yields remarkable high-resolution reduction of time-side lobe and continuous scattering pattern of signal has been achieved. 3D representation of CPM shown in Figure 10f gives clear vision of the targets.

\section{CONCLUSIONS}

The time-domain response of IFFT and MUSIC have been combined to obtain super-resolution and high-precision receiving signal level. The proposed CPM could successfully resolve vertically separated targets up to $2 \mathrm{~cm}$ at $800 \mathrm{MHz}$ frequency bandwidth in water medium, as shown in laboratory experiment. The field experiment and laboratory experiment results show the remarkable reduction of time-side lobes and natural clutter. Moreover, CPM could successfully demonstrate the continuous scattering pattern of the radar signal that is realized from 2D and 3D images of both laboratory and field experiment. From the simulation results, it is concluded that CPM has higher resolution than other conventional signal processing methods due to the effect of MUSIC and also the precision of receiving signal level is high due to the effect of IFFT. Further, the SNR analysis results show that the proposed method is robust from the point of view of noise if the value of $M$ is increased during the smoothing process. These are the major achievement of this research work. The proposed method could be practically implemented to detect closely buried water pipes, gas pipes, cables, and even antipersonnel and antitank land mines.

\section{ACKNOWLEDGMENTS}

The authors would like to thank the Koden-Electronics Company, Yamanashi Prefecture, Japan, for providing its research field for experimental purpose. We would like to thank Dr. Kazuo Yamamoto at Electronic Navigation Research Institute (ENRI), Tokyo, Prof. Christian Pichot at LEAT, University of Nice-Sophia, France, and Mr. Michael Cashen at the University of Electro-Communications, Tokyo, for their suggestions and support. The authors would like to thank the reviewers for providing comments and suggestions, which have greatly improved the paper. 


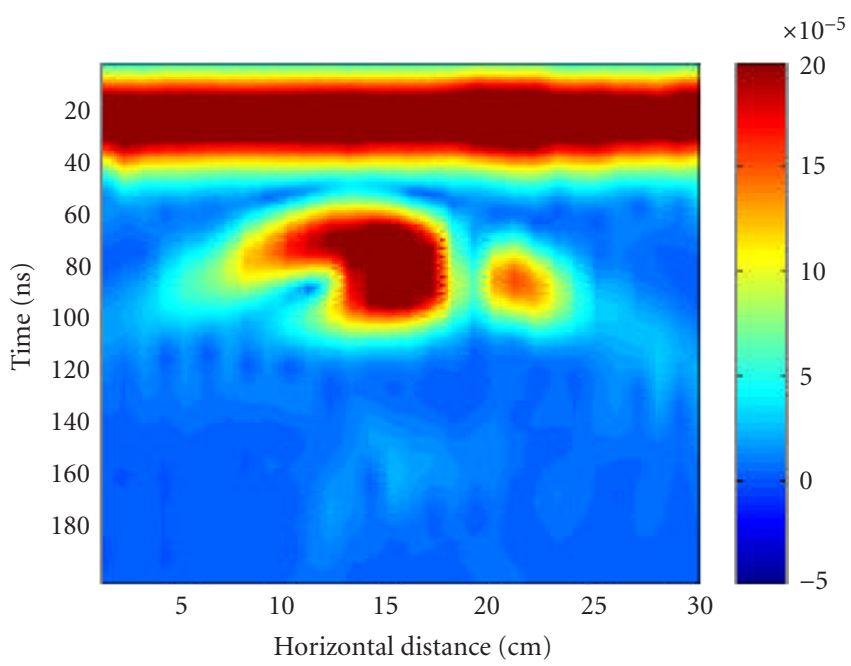

(a) 2D representation of IFFT processing.

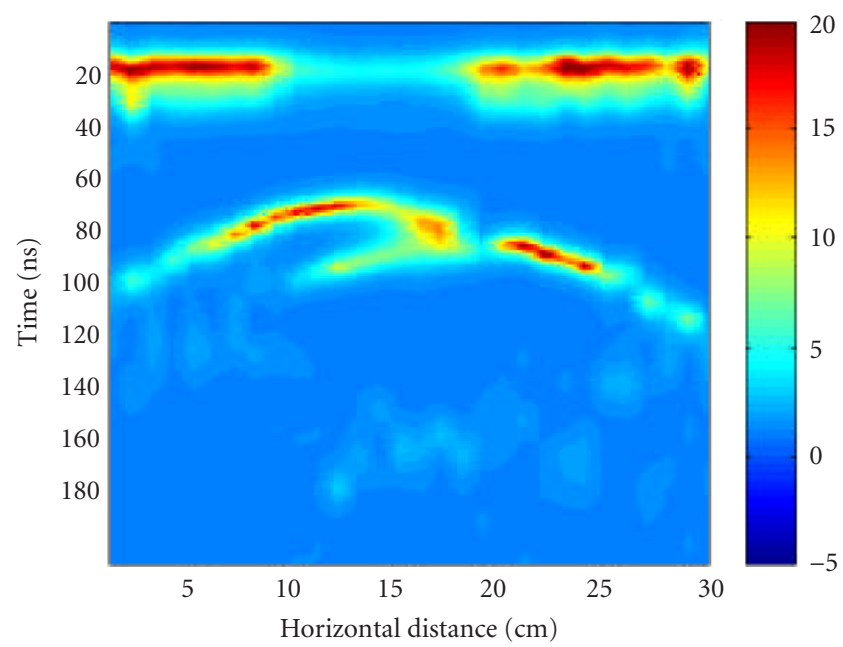

(c) 2D representation of MUSIC processing.

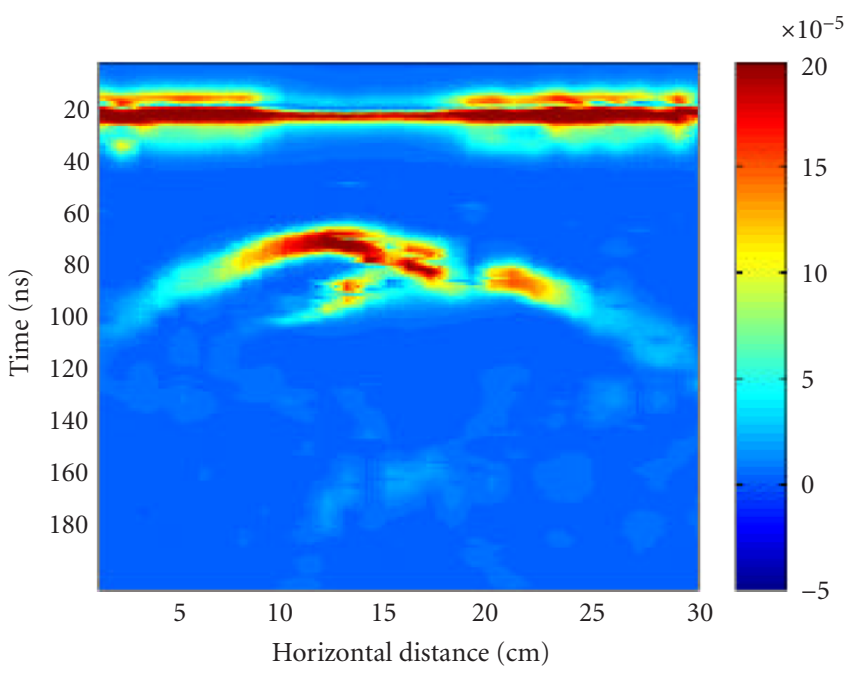

(e) $2 \mathrm{D}$ representation of CPM processing.

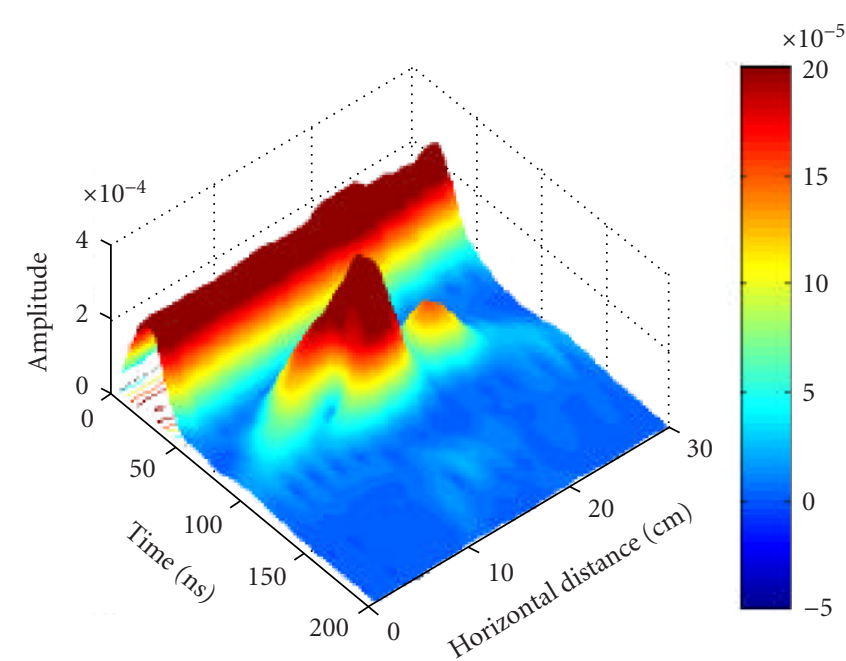

(b) 3D representation of IFFT processing.

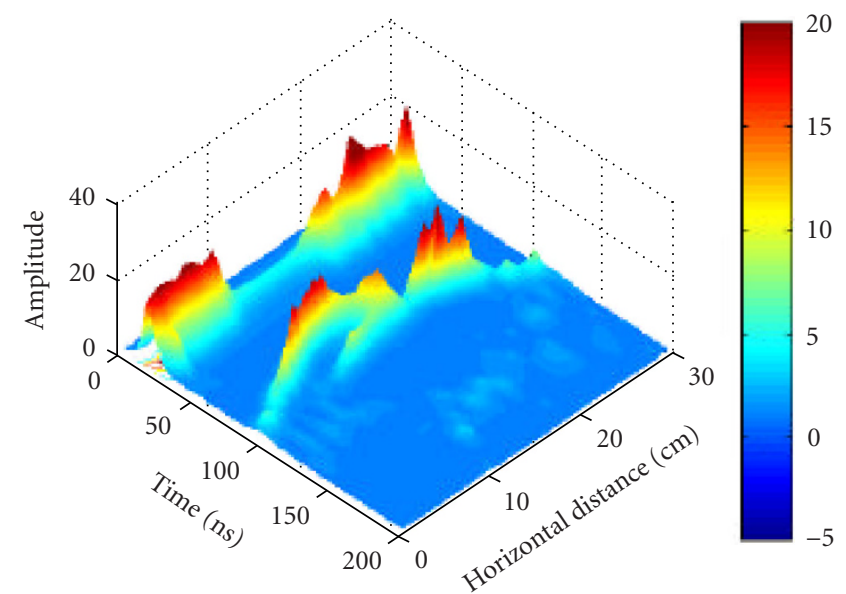

(d) 3D representation of MUSIC processing.

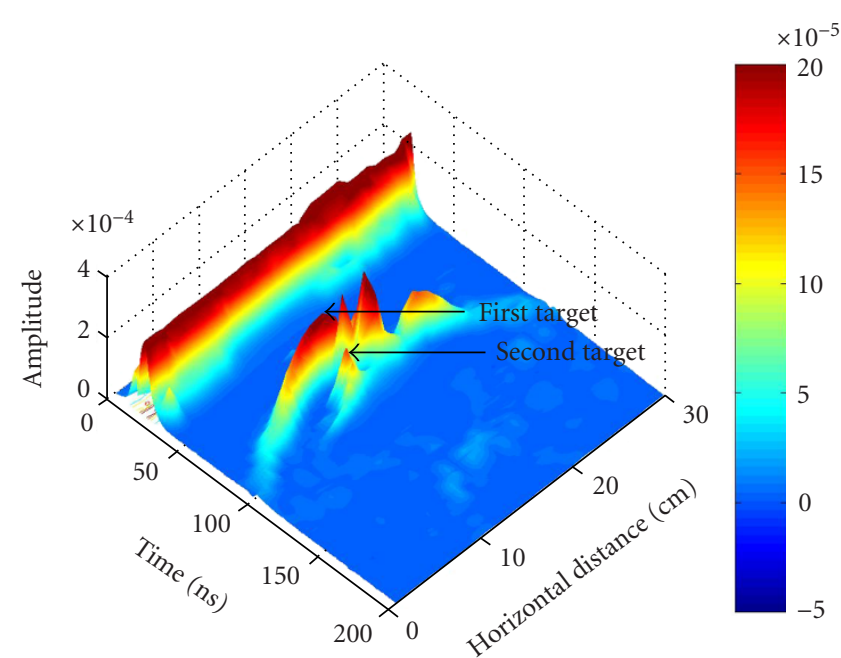

(f) 3D representation of CPM processing.

FIGURE 10: 2D and 3D representations of IFFT, MUSIC, and CPM processing of laboratory experiment data with bandwidth $=800 \mathrm{MHz}$, $M=30$, and $L=150$. 


\section{REFERENCES}

[1] I. Arai, Y. Tomizawa, and M. Hirose, "Pulse compression subsurface radar," IEICE Transactions on Communications, vol. E83-B, no. 9, pp. 1930-1937, 2000.

[2] Y. Tomizawa and I. Arai, "Subsurface radar using a coded signal without time-sidelobes," Journal of Remote Sensing Society of Japan, vol. 21, no. 5, pp. 423-430, 2001.

[3] W. R. Scott Jr., J. S. Martin, and G. D. Larison, "Experimental model for a seismic landmine detection system," IEEE Transactions on Geoscience and Remote Sensing, vol. 39, no. 6, pp. 1155-1164, 2001.

[4] J. I. Halman, K. A. Shubert, and G. T. Ruck, "SAR processing of ground-penetrating radar data for buried UXO detection: results from a surface-based system," IEEE Trans. Antennas and Propagation, vol. 46, no. 7, pp. 1023-1027, 1998.

[5] J. Capon, "High resolution frequency-wave number spectrum analysis," Proc. IEEE, vol. 57, pp. 1408-1418, August 1969.

[6] A. Yoshizawa and T. Iwasaki, "Image reconstruction in optical frequency-domain reflectometry by means of maximum entropy method-Fourier transform combination," IEICE Trans., vol. J76-C-I, no. 6, pp. 215-221, 1993.

[7] R. Roy and T. Kailath, "ESPRIT-Estimation of signal parameters via rotational invariance techniques," IEEE Trans. Acoustics, Speech, and Signal Processing, vol. 37, no. 7, pp. 984-995, 1989.

[8] R. O. Schmidt, "Multiple emitter location and signal parameter estimation," IEEE Trans. Antennas and Propagation, vol. 34, no. 3, pp. 276-280, 1986.

[9] J. F. Evans, J. R. Johnson, and D. F. Sun, "Application of advanced signal processing techniques to angle of arrival estimation in A.T.C. navigation and surveillance systems," Tech. Rep. 582, MIT Lincoln Lab, Lexington, Mass, USA, June 1982.

[10] T.-J. Shan, M. Wax, and T. Kailath, "On spatial smoothing for direction-of-arrival estimation of coherent signals," IEEE Trans. Acoustics, Speech, and Signal Processing, vol. 33, no. 4, pp. 806-811, 1985.

[11] R. T. Williams, S. Prasad, A. K. Mahalanabis, and L. H. Sibul, "An improved spatial smoothing technique for bearing estimation in a multipath environment," IEEE Trans. Acoustics, Speech, and Signal Processing, vol. 36, no. 4, pp. 425-432, 1988.

[12] M. I. Skolnik, Radar Handbook, McGraw-Hill, NY, USA, 2nd edition, 1990.

[13] H. Yamada, M. Ohmiya, Y. Ogawa, and K. Itoh, "Superresolution techniques for time-domain measurements with a network analyzer," IEEE Trans. Antennas and Propagation, vol. 39, no. 2, pp. 177-183, 1991.

[14] T. Akiyama, T. Yamaoka, and N. Hamada, "2-d direction-ofarrival estimation using a linear interpolation technique for circular array," IEICE Transactions on Communications, vol. E84-B, no. 9, pp. 215-221, 2001.

[15] J.-S. Jeong, K. Sakaguchi, K. Araki, and J. Takada, "Generalization of MUSIC using extended array mode vector for joint estimation of instantaneous DOA and angular spread," IEICE Transactions on Communications, vol. E84-B, no. 7, pp. 1781$1789,2001$.

[16] T. Yamakura, H. Yamada, and Y. Yamaguchi, "Resolution improvement of the MUSIC algorithm utilizing two differently polarized antennas," IEICE Transactions on Communications, vol. E79-B, no. 12, pp. 1827-1832, 1996.

[17] K.-T. Kim, D.-K. Seo, and H.-T. Kim, "Efficient radar target recognition using the MUSIC algorithm and invariant features," IEEE Trans. Antennas and Propagation, vol. 50, no. 3, pp. 325-337, 2002.

[18] S. M. Shrestha, I. Arai, and T. Miwa, "Signal processing of ground penetrating radar combining FFT and MUSIC algo- rithm for high resolution," Tech. Rep. IEICE SANE2000, SAT2000-130, Tokyo, Japan, 2001/2002.

[19] S. M. Shrestha, I. Arai, T. Miwa, and Y. Tomizawa, "Signal processing of ground penetrating radar using super resolution technique," in IEEE Radar Conference, pp. 300-305, Atlanta, Ga, USA, May 2001.

Shanker Man Shrestha received his B.S. degree in electrical and electronic engineering from the Bangladesh University of Engineering and Technology (BUET), Dhaka in 1993, and his M.E. degree in electronic engineering from the University of ElectroCommunications (UEC), Tokyo, Japan in 2001. He is currently working towards the Ph.D. degree at the UEC. From 1994 to 1998, he worked as a Communications En-

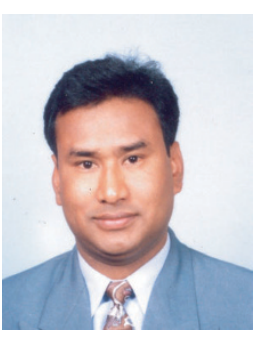
gineer in Transmission Planning, and Satellite Communication Department, Nepal Telecommunications Corporations (NTC), Kathmandu, Nepal. From 1998 to 1999, he was a research student at the UEC, Tokyo, Japan. His research interests include superresolution signal processing algorithm, ground penetrating radar (GPR), FMCW radar, SAR (synthetic aperture radar), mobile and satellite communications, and so on. His recent research activities include the development of GPR for the land mine detection, application of MUSIC algorithm in GPR, millimeter wave radar to detect the helicopter obstacles. He is a student member of the IEEE, IEICE, and Remote Sensing Society of Japan.

Ikuo Arai was born in Tochigi Prefecture, Japan in 1941. He received his B.S. and M.S. degrees from the University of ElectroCommunications, Tokyo, Japan in 1965 and 1967, respectively, and his Ph.D. degree in electrical engineering from the University of Tokyo in 1986. Since his graduation, he has been working as a faculty member at the University of Electro-Communications, Tokyo, Japan. Currently, he is a Professor in

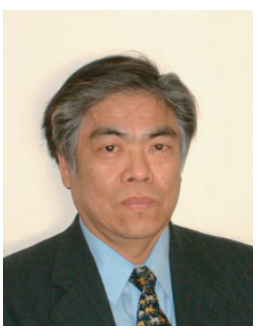
the Department of Electronics Engineering. He has been working in ground penetrating radar for 25 years. He is the Author and Coauthor of several papers and books. His research interests include medical electronics, ground penetrating radar, and highresolution radar. He is a member of the Remote Sensing Society of Japan, IEICE, and Archaeological Prospection Society of Japan. 